\title{
Die Krise der EU und die Stärke einer deliberativen Integrationstheorie
}

\section{Einleitung}

Die Europäische Union ist in der Krise. Und das ist gut so, ist man versucht zu sagen. Bekanntlich steht der Begriff der Krise nicht nur für das Ende des Alten, sondern ebenfalls für den Beginn des Neuen. Und ein Neubeginn ist in Europa dringend geboten. Christopher Daase hat den Teilnehmern an diesem Symposium drei Fragen mit auf den Weg gegeben: Wie lässt sich die Krise der EU aus theoretischer Sicht erklären, warum war die Integrationstheorie nicht in der Lage, die Krise zu antizipieren, und ist die Integrationstheorie daher letztlich obsolet geworden? Ich werde die Fragen der Reihe nach beantworten und dabei zu zeigen versuchen, dass ein deliberativer Supranationalismus - im Gegensatz zu manchen anderen Theorien - sehr wohl in der Lage ist, die gegenwärtige Krise zu erklären sowie Vorhersagen über zukünftige Krisen zu formulieren. Integrationstheorie ist daher keineswegs obsolet geworden, sondern vielmehr von hoher wissenschaftlicher und politischer Aktualität.

Der deliberative Supranationalismus ist ursprünglich als regulative Idee im Kontext einer Untersuchung des europäischen Ausschusswesens entstanden (Joerges/ Neyer 1997) und hat sich seitdem aber als eigenständiger theoretischer Zugang zur Analyse der europäischen Integration einen festen Platz in der theoretischen Landschaft erobert. ${ }^{1}$ Die Kernidee liegt darin, dass die Fähigkeit eines nicht-hierarchisch aufgebauten Gemeinwesens zum einvernehmlichen Handeln unter den Bedingungen gesellschaftlicher Pluralität nur als kommunikativer und inklusiver Prozess und unter Zuhilfenahme von kodifizierten Rechtsnormen gedacht werden kann. Übertragen auf die Union bedeutet dies, dass politische Einigung als rechtsbasierte Mehrebenendeliberation unter Einschluss der europäischen Institutionen, der Mitgliedsstaaten und ihrer Gesellschaften zu konzeptualisieren ist.

Der deliberative Supranationalismus versteht sich als Alternative zum Intergouvernementalismus und Neofunktionalismus. Im Gegensatz zum Intergouvernementalismus betont der deliberative Supranationalismus die zentrale Rolle europäischen Rechts als Verständigung forderndes und förderndes Medium. Gleichzeitig setzt sich der deliberative Supranationalismus vom Funktionalismus ab. Es gibt keinen Automatismus der Integration, der sich unabhängig von politischen Entscheidungsund Verständigungsprozessen vollziehen würde, sondern lediglich rechtsbasierte Entscheidungsprozesse, die zu einem Mehr, genauso aber auch zu einem Weniger an Integration führen können. Der wesentliche Grund hierfür liegt in der demokrati-

1 Für einen Überblick über den Stand der Literatur siehe Schmalz-Bruns (1999), Joerges (2000), Neyer (2003), Eriksen/Fossum (2004). 
schen Konstitution der Mitgliedsstaaten, die ihre Regierungen nötigt, sich für verfolgte Politiken öffentlich zu rechtfertigen. Dort wo diese Rechtfertigung unterbleibt oder als unglaubwürdig wahrgenommen wird, entsteht latenter oder faktischer Widerstand.

\section{Warum ist die EU in der Krise?}

Für die Erklärung der aktuellen Krise der Union ist der deliberative Supranationalismus ganz offensichtlich relevant. Nach wie vor ist die Europäische Union in weiten Teilen ein technokratisch-intergouvernementales Regime, in dem die wichtigsten Entscheidungen von den Regierungen hinter verschlossenen Türen und unter Ausschluss breiterer gesellschaftlicher Kreise getroffen werden (Neyer/Schroeter 2005). Es ist zwar zutreffend, dass der Ministerrat gelegentlich öffentlich tagt und ebenfalls ist es richtig, dass die Kompetenzen des Parlamentes über die Zeit immer weiter ausgedehnt wurden. Gleichzeitig aber wurde der Europäische Rat in seiner Rolle als Agenda setzende Institution bestärkt und dem Parlament die Kompetenz verweigert, eigenständig die politische Agenda der EU bestimmen zu können. Das Parlament ist daher letztlich lediglich in seiner Funktion gestärkt worden, den Mitgliedsstaaten dabei zu helfen, ihre im Europäischen Rat gefundene Agenda umzusetzen. Die wichtigsten politischen Entscheidungen und Weichenstellungen der europäischen Politik finden aber nicht im Parlament - oder auch nur zwischen Parlament und Ministerrat -, sondern hinter den verschlossenen Türen des Europäischen Rates statt. Auch der hoch gelobte Verfassungskonvent war letztlich wenig mehr als eine beratende Versammlung von Honoratioren und Berufseuropäern. Die Beratungen des Konvents erfolgten zudem im Schatten der folgenden Regierungskonferenz und waren daher von vornherein von der Notwendigkeit geprägt, nur solche Ergebnisse zu produzieren, die für die mitgliedsstaatlichen Regierungen akzeptabel wären (vgl. Magnette/Nicolaides 2004).

Es kann daher auch nicht überraschen, dass fast alle der wirklich wichtigen politischen Weichenstellungen der letzten Jahre auf rein intergouvernementale Beschlüsse zurückgehen: Die mitgliedsstaatlichen Exekutiven handelten sowohl die Wirtschafts- und Währungsunion mit ihrem Stabilitätspakt als auch die Erweiterung der EU um die zehn osteuropäischen Staaten unter sich aus. Der Umbau der Union in einen Raum der Sicherheit und des Rechts und die hiermit einhergehende Ausdehnung gouvernementaler Kompetenzen wurde den europäischen Gesellschaften vom Europäischen Rat als eine notwendige Reaktion auf die Terrorismusgefahr präsentiert und ebenso per intergouvernementaler Deklaration verkündet - wie der Ausbau der militärischen Komponente der GASP und die hiermit einhergehende Militarisierung der europäischen Außenpolitik.

Auch die Reaktion der Europäischen Union auf die Herausforderung der Globalisierung ist alles andere als unkontrovers. Globalisierungsbezogene Politik der Union beschränkt sich heute weitestgehend auf Maßnahmen zur Förderung von Wettbewerbsfähigkeit und neuen Technologien und hat keinerlei Sensibilität für die sozia- 
len Ängste ihrer Bürger. Die Entwicklung einer europäischen Beschäftigungsstrategie ist über unverbindliche Empfehlungen nicht hinausgekommen. Aufgrund der einseitigen Konzentration der EU-Politik auf die Beförderung der negativen Integration gilt der Begriff der Europäisierung für viele heute schon als Synonym für die sozialen Verwerfungen der Globalisierung. Die Osterweiterung steht im Bewusstsein vieler Menschen daher auch nicht für die historische Überwindung der Teilung des Kontinents, sondern für erhöhten Wettbewerbsdruck und weiteren Arbeitsplatzverlust. Aus der ehemaligen Hoffnung auf eine Antwort auf die Globalisierung ist das trojanische Pferd der Globalisierung geworden. Es geht hier nicht darum, einzelne dieser Politiken inhaltlich zu kritisieren. Für alle lassen sich gute inhaltliche Gründe vorbringen. Das zentrale Problem liegt tiefer. Mit der Osterweiterung, dem Stabilitätspakt, der Militarisierung der GASP und der Globalisierung hat die Union die »Nichtsichtbarkeitsschwelle « überschritten und den ehemals so wirkungsvollen permissiven Konsens in Frage gestellt.

Die EU ist relevant und politisch erwachsen geworden. Damit aber hat sie sich automatisch auch mit wachsender Kritik auseinander zu setzen und muss auf sie eingehen. In der mitgliedsstaatlichen Demokratie geschieht diese Auseinandersetzung alläglich in den Medien und regelmäßig über Regierungserklärungen. In der EU fehlt sie hingegen weitestgehend. Weder gibt es eine institutionalisierte Opposition noch publikumswirksame Foren der Auseinandersetzung mit abweichenden Meinungen. Die EU ist faktisch zu einer Form der organisierten Unverantwortlichkeit verkommen, in der Konsense von allen Organen der EU sowie allen Mitgliedsstaaten mitgetragen werden und doch keiner so recht verantwortlich ist. Die alltägliche Rechtfertigung für Politikinhalte und die Auseinandersetzung mit Kritik hat aber eine außerordentlich wichtige Funktion. Nur sie kann gewährleisten, dass potenziell konstruktive Kritik verarbeitet und nicht zu destruktiver Kritik am System als solchem wird. Die Ablehnung des Verfassungsvertrages ist in dieser Perspektive weder ein rein innenpolitisches Problem noch bloß auf Defizite des Dokumentes zurückzuführen. Beides mag zwar durchaus zur Ablehnung des Verfassungsvertrages beigetragen haben, bildet aber kaum den Kern des Problems. Der Kern des Problems liegt vielmehr in den Strukturen der Europäischen Union und ihrer mangelnden Fähigkeit zur konstruktiven Auseinandersetzung mit und Einbindung von Opposition.

\section{Warum konnte die Integrationstheorie die Krise nicht vorhersehen?}

Die Antwort auf diese Frage liegt auf der Hand. Der ganz überwiegenden Mehrheit der Integrationstheorien scheint der simple Umstand entgangen zu sein, dass es in Europa politische Gesellschaften mit einer ausgeprägten Fähigkeit zur Artikulation ihrer Präferenzen gibt. Es gibt zwar vielleicht keine eine »europäische« Gesellschaft, aber dafür inzwischen 25 nationale Gesellschaften, die sich allesamt - zumindest ab und zu - mit Europa beschäftigen. Im Gegensatz zur analytischen Demokratietheorie, die ohne den Begriff der Gesellschaft schlechterdings nicht gedacht werden kann 
(das gilt für die normative Demokratietheorie natürlich umso mehr), ist der Begriff der Gesellschaft die Integrationstheorie noch immer ein Fremdwort. Der (Neo-) Funktionalismus ist eine geradezu a-demokratische Theorie, die Integrationsprozesse auf Automatismen reduziert und Gesellschaft nur insoweit zur Kenntnis nimmt, als hier Agenten weiterer Integration identifiziert werden können (vgl. etwa Corbey 1995; Stone Sweet/Sandholtz 1997). Der Begriff der Gesellschaft selbst ist dem Neofunktionalismus hingegen fremd. Es kann daher auch kaum verwundern, wenn Neofunktionalisten von der Ablehnung des Verfassungsvertrages überrascht sind, die resultierenden ökonomischen und politischen Ineffizienzen beklagen und eine Wiederholung der Referenden fordern.

Nicht viel besser steht hier der Intergouvernementalismus da. Zumindest in seiner liberalen Ausprägung ist er sich zwar durchaus der Anbindung von Regierungen an vorherrschende gesellschaftliche Präferenzen bewusst, reduziert die Funktion von Regierungen aber auf die Darstellung dominanter Gewerkschafts- oder Unternehmensinteressen (vgl. Moravcsik 1998). Gesellschaft erscheint hier in einer korporatistisch verengten Sichtweise, die nur institutionalisierte Interessen kennt und diffuse Interessenlagen sowie nicht-organisierte gesellschaftliche Meinungen weitestgehend ausblendet. Während die klassische Demokratieanalyse erwarten ließe, dass jede Regierung schon aus Gründen des Machterhaltes immer versuchen muss, breite Gesellschaftskreise zu erreichen und »volksparteiliche« Politik zu gestalten, ist die Regierung im liberalen Intergouvernementalismus offensichtlich ausschließlich für die Wünsche gut organisierter Interessengruppen offen. Die demokratische Öffentlichkeit, vor der Politiken im diskursiven Prozess zu rechtfertigen sind, taucht als analytische Kategorie hingegen überhaupt nicht auf. Von einer Integrationstheorie, die in derart gravierender Weise die gesellschaftlichen Grundlagen der Europäischen Union ausblendet, kann in der Tat kaum erwartet werden, dass sie gesellschaftlich induzierte Krisen vorhersieht.

\section{Ist die Integrationstheorie obsolet geworden?}

Vor dem Hintergrund der im Bisherigen angestellten Kritik ist die dritte Frage nach der Obsoleszenz der Integrationstheorie abgewogen zu beantworten. Wenn es zutreffend ist, dass die politischen Gesellschaften Europas sich inzwischen ihren Platz im politischen Geschäft der Union erobert haben und Regierungen es sich kaum noch leisten können, über die vorherrschenden Meinungen hinwegzugehen, dann sind zumindest alle solchen Theorien überholt, die den politischen Gesellschaften Europas keinen eigenständigen analytischen Ort einräumen. Obsolet geworden wäre dann ein korporatistisch verkürzter Intergouvernementalismus ebenso wie ein technokratischer Neofunktionalismus.

Beide mögen zwar durchaus noch erklärendes Potenzial haben, wenn wir uns mit der politischen Vergangenheit der EU beschäftigen, uns im Arkanbereich der europäischen Bürokratie bewegen oder uns einzelne Entscheidungsprozesse des Europäischen Rates anschauen. Nach wie vor ist es sicherlich auch zutreffend, dass 
nur die wenigsten Entscheidungen in der Union deliberativen und inklusiven Verfahren folgen. Der Europäische Rat ist und bleibt wohl auf weitere Zeit die dominante Institution in der Union und das Parlament wird in absehbarer Zukunft kaum eigenständige Agendasetzungsmacht gewinnen.

Ihre Grenzen haben Intergouvernementalismus und Neofunktionalismus hingegen dort, wo die politischen Gesellschaften Europas ins Spiel kommen. In allen Fällen, in denen breitere gesellschaftliche Kreise eine Teilhabe an europäischen Entscheidungen verlangen - sei dies bei der Zulassung britischen Rindfleischs auf den europäischen Markt oder bei der Annahme einer Verfassung für Europa ist - haben deliberative Ansätze ihre Stärken. Politische Entscheidungen müssen dann im Einvernehmen zwischen Regierungen, europäischen Institutionen und europäischen Gesellschaften getroffen werden, wenn nicht Konfliktpotenzial aufgebaut und künftige Krisen vorprogrammiert werden sollen. Im Einzelfall lassen sich die politischen Meinungen auch breiter gesellschaftlicher Kreise übergehen. Wenn es allerdings der Regelfall in der Union bleibt, dass Entscheidungsprozesse jenseits gesellschaftlicher Rechtfertigung und ohne wirkliche Auseinandersetzung mit Betroffenen stattfinden, dann ist zu erwarten, dass die Krise der Union zum Dauerzustand wird. Europa ist in den letzten zwanzig Jahren zumindest im Hinblick auf seine Politikinhalte erwachsen geworden. Es wird Zeit, dass sich seine Entscheidungsverfahren dem anpassen. Der wichtigste Schritt auf diesem Weg ist, der Opposition gegen spezifische europäische Politiken einen institutionellen Platz einzuräumen, damit die Opposition gegen den europäischen Weg ein Randphänomen bleibt. Wer die Deliberation und damit auch die kritische Opposition in Europa verhindert, droht hingegen das europäische Projekt selbst zu gefährden.

\section{Literatur}

Corbey, Dorette 1995: Dialectical Functionalism: Stagnation as a Booster of European Integration, in: International Organization 49: 2, 253-284.

Eriksen, Erik O./Fossum, John 2004: Europe in Search of Legitimacy: Strategies of Legitimation Assessed, in: International Political Science Review 25: 4, 435-459.

Joerges, Christian 2000: Transnationale »deliberative Demokratie« oder »deliberativer Suprantionalismus «? Anmerkungen zur Konzeptualisierung legitimen Regierens jenseits des Nationalstaats bei Rainer Schmalz-Bruns, in: Zeitschrift für Internationale Beziehungen $7: 1,145-161$.

Joerges, Christian/Neyer, Jürgen 1997: From Intergovernmental Bargaining to Deliberative Political Processes: The Constitutionalisation of Comitology, in: European Law Journal 3: 3, 273-299.

Magnette, Paul/Nicolaides, Kalypso 2004: The European Convention: Bargaining in the Shadow of Rhetoric, in: West European Politics 27: 3, 381-404.

Moravcsik, Andrew 1998: The Choice for Europe: Social Purposes and State Power from Messina to Maastricht, Ithaca, NY.

Neyer, Jürgen 2003: Discourse and Order in the EU: A Deliberative Approach to Multi-Level Governance, in: Journal of Common Market Studies 41: 4, 687-706.

Neyer, Jürgen/Schroeter, Michael 2005: Deliberative Europe and the Rejected Constitution (Abschlusskonferenz des CIDEL-Projektes, Europäisches Hochschulinstitut Florenz, 22. - 24. 9.2005). 
Schmalz-Bruns, Rainer 1999: Deliberativer Supranationalismus. Demokratisches Regieren jenseits des Nationalstaats, in: Zeitschrift für internationale Beziehungen 6: 2, 185-244.

Stone Sweet, Alec/Sandholtz, Wayne 1997: European Integration and Supranational Governance, in: Journal of European Public Policy 4: 3, 297-317. 